\title{
Research Methods of the Waterway Dimension Which May Be Achieved by Long Stream Segment Regulation
}

\author{
Wanli LIU ${ }^{1}$, a , Xiaofei LIU¹, Kejun PING ${ }^{1}$, Pengfei LIU1, Ying LU², Fei HAN², \\ ${ }^{1}$ Key Laboratory of Engineering Sediment of Ministry of Communications, Tianjin Research Institute \\ for Water Transport Engineering, Tianjin 300456, China \\ atjtgliuwanli@163.com \\ ${ }^{2}$ Changjiang Waterway Bureau, Wuhan 430010, China
}

Keywords: research Methods; waterway dimension; Long Stream Segment

Abstract. In the past, waterway regulation generally proposed optimized project schemes and the corresponding regulation measures according to the waterway dimension of the design requirements, upon the requirements of satisfaction of the standard waterway dimension. However, the issue of the dimension falling within reasonable limits was not considered. This article proposes five research methods about the waterway dimension which long stream segment regulation may achieve as well as specific computational methods.

\section{Introduction}

The design waterway dimension generally refers to the navigation depth, navigational width and curvature radius which are calculated with the lowest design navigation water level. Ascertaining the design waterway dimension is a key step in planning and designing inland river waterway projects. Its accuracy indirectly influences the success or failure of regulation project, efficiency and development of water transport business. Currently, there are five methods for ascertaining the waterway dimensions domestically. They are economic waterway dimension, ascertaining the waterway dimension with selected ships, inland river navigation standard, waterway dimension which the regulation may achieve and method combining canalization and regulation. The waterway dimension which the regulation may achieve needs detailed materials of terrain, geology, hydrology, sediments and riverbed composition of the local rivers. The possible potential reasonable limit dimension of the local river can only be ascertained through river evolution analysis and mathematical and physical model arguments. This article will examine the long stream segment of the river in western mountain area and seek for research methods of the maximum waterway dimension which the mountain area river can achieve through regulation, which is of great significance for guiding the passing capacity improvement of the long stream segment waterway.

The "waterway dimension" problem involves many influential factors, such as natural conditions (hydrology, terrain and geology), environmental conditions, regional economic status, developmental prospects and etc. The so-called "waterway dimension which may achieves" requires measurement criteria and limitative conditions; otherwise there will be no definite solution. According to regional economic development level and requirements, it plans to have the following formulations: 
(1) The optimum waterway dimension of the natural navigation depth or hydraulic permissible navigation depth;

(2) The hydraulic optimum waterway dimension;

(3) The optimum waterway dimension under the condition of a certain regulation project quantity;

(4) The waterway dimension when the regulation project quantity is the minimum under a certain annual freight volume;

(5) The optimum waterway dimension under the condition of "limit" increased depth.

The first two kinds are low levels of waterway regulation, which are applicable to the circumstance in which the regional economy is not developed and the annual volume of freight is small; The latter three kinds are medium and high levels of waterway regulation, which are applicable to conditions involving a relatively developed regional economy and a high annual freight volume.

They can be collectively referred to as the problem of "the optimum waterway dimension of long stream segment regulation".

\section{The Optimum Waterway Dimension of the Natural Navigation Depth}

(1) Draw the flow quantity assurance rate curve according to the perennial daily average flow quantity;

(2) Use the mathematical model to ascertain the water level (water depth) of each shoal along the waterway under different flow quantities for the medium and dry seasons;

(3) Draw the relationship curve between the navigation depth and flow quantity of each shoal. As shown in Figure 1, its lower envelop boundary is the relationship between the natural navigation depth and the flow quantity of the long stream segment. It is also possible to plot directly the relationship between the minimum navigation depth of the shoal group and flow quantity under the flow quantity of each class.

(4) The corresponding design navigation depth can be ascertained according to the design assurance rate; or ascertain the corresponding assurance rate according to the design navigation depth. When the product of the design navigation depth and design assurance rate is the maximum, it means that the annual volume of freight is the maximum and it is the optimum waterway dimension.

This waterway dimension is the natural waterway dimension when the waterway is in its initial condition, which doesn't make use of the navigation resources of the river effectively. Although no development capital is invested, it is a waste of the resource.

\section{The Hydraulic Optimum Waterway Dimension}

The hydraulic optimum waterway dimension is the waterway dimension when the regulation project is the minimum, under the condition of the maximum usage of the water depth of the shoal. Under this dimension, the increased depth (i.e. the navigation-obstructing depth) and the underkeel water depth are both minimum.

Calculate the different flow quantity $\mathrm{Q}$ and different navigation depth $\mathrm{h}-\mathrm{ht}$ (the negative value is the navigation-obstructing depth, and the positive value is the underkeel water depth). And carry out positive and negative accumulation respectively, i.e. $\pm \Sigma(\mathrm{h}-\mathrm{ht})$. Take $\mathrm{Q}$ as the parameter, and draw the relationship between $\pm \Sigma(h-h t)$ and ht as it is shown in Figure 2. Figure 2 is ascertained 
according to the specific numerical values of curves in Figure 1. In the figure, the four levels of flow quantities have four points of intersection, i.e. 1 4. Among them, the navigation-obstructing water depth and underkeel water depth of point 2 are both minimum. The corresponding waterway water depth and assurance rate (or Q) to this point are the hydraulic optimum waterway dimension.

Figure 2 shows that, the navigation-obstructing depths and underkeel depths of points 1 4 are equal to one another. That is to say, the corresponding navigation depth is the average water depth of the shoal group under such a flow quantity. Therefore, the hydraulic optimum navigation depth is the corresponding average water depth to the minimum mean square error of the average water depth of the shoals under different flow quantities. The minimum mean square error of the a verage water depth of the shoals is:

$$
\begin{gathered}
\sigma_{\mathrm{m} \mathrm{in}}=\min \frac{\sqrt{\left(\frac{\sum(h-\bar{h})^{2}}{n}\right)}}{n} \\
-{ }_{h=\Sigma} h / n
\end{gathered}
$$

In the equation, $\bar{h}$ is the average water depth of the shoals under a certain flow quantity; hi is the minimum water depth of the itch shoal under different flow quantities; $\mathrm{n}$ is the number of shoals.

\section{The Optimum Waterway Dimension under the Condition of a Certain Regulation Project Quantity}

A certain waterway increased depth has a certain regulation project quantity. Figure 2 shows that, when the total waterway increased depth is $\mathrm{A}$, there are countless combinations of ht and $\mathrm{Q}$, i.e. combinations of ht and P. For example, the combination of ht 4 and Q2 is one of them. Because ht is direct proportional to the ship tonnage, when the ht is large, the volume of freight of a single ship is also large. The annual volume of freight is direct proportional to htP. In the countless combinations of ht and $\mathrm{P}$, there must be a group of htP which is the maximum. Among them, the combination of ht and $\mathrm{P}$ corresponding to the $\max (\mathrm{htP})$ is the optimum waterway dimension.

\section{The Waterway Dimension under the Condition of a Certain Annual Volume of Freight When the Regulation Project Quantity is Minimum}

According to a certain waterway increased depth, obtain max (htP) and the corresponding ht. Draw the relation curve of $\mathrm{ht} \sim$ the $\max (\mathrm{Pht})$, and in it, the waterway dimension ht and $\mathrm{P}$ corresponding to the set $\mathrm{Pht}$ are the waterway dimension under the condition of minimum regulation project quantity.

\section{The Optimum Waterway Dimension Under "Limit Increased Depth"}

The so-called "limit increased depth" refers that, provided there is a shoal of the group shoals, the increased depth of which achieves the "limit", it is not possible or not allowed to deepen further. Such circumstances include:

(1)Upstream project restriction, for example, diversion works and etc;

(2)Embankment and flood control safety restriction;

(3)The downstream shoal regulation has serious influence on the upstream dangerous shoals or un-shoal stream segments; 
(4)The regulation project quantity is huge and etc. Therefore, the so-called "limit" is relative and has hardly strict limit.

The optimum waterway dimension under such condition is similar to the above-mentioned Chapter 3, with the only modification of "a certain waterway increased depth" to "limit increased depth".

According to the characteristics of each dangerous shoal of the whole river and the relationships between the adjacent dangerous shoals, differentiate the three circumstances of backwater increased depth, dredging increased depth, and increased depth with a combination of dredging and lifting. Classify the interrelated dangerous shoals into one group called shoal group. Divide the whole river into several independent shoal groups. Research the regulation measurements of each independent shoal group, and build up the relationship between increased depth and project quantity. According to the control shoal, determine the "limit" condition. Because the relationship between the water level and flow quantity will change after the shoal project, and the upper and lower shoals may be of mutual influence on each other, we need to repeat trial calculations and adjustments over again when building the relationship between the increased depth and the project quantity. Each independent shoal group can be carried out respectively in order to decrease the workload.

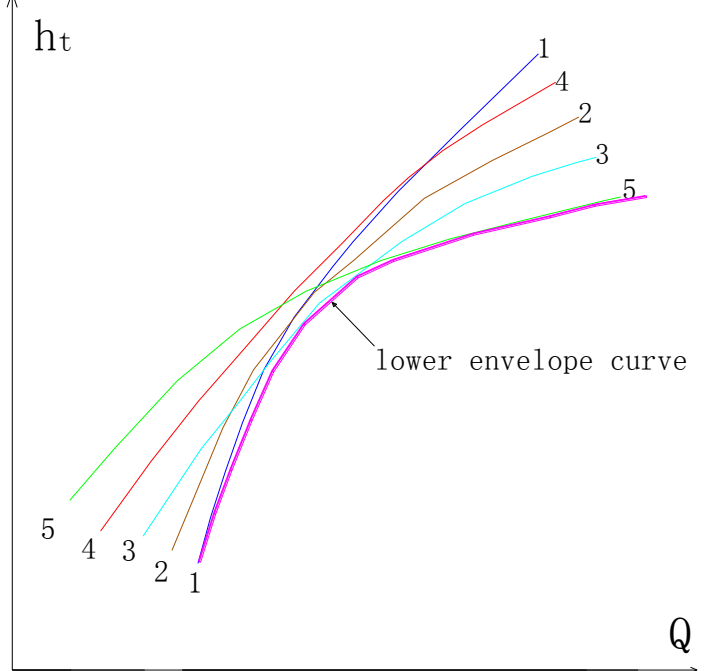

Fig.1 Sketch of the Relationship between Water Depth and Flow Quantity of Each Shoal in the Long River Segment

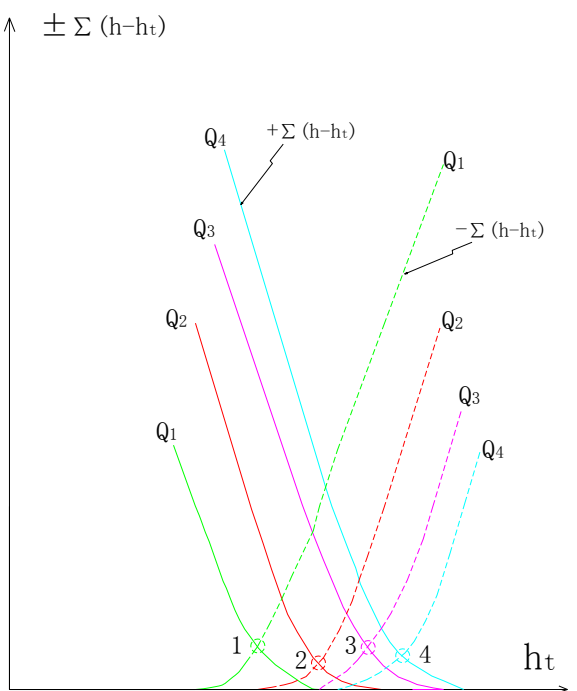

Fig.2 Graph Showing the Relationship between the Accumulated Navigation-obstructing Depth and under keel Depth of the Shoal 


\section{Conclusions}

The "waterway dimension" problem involves many influential factors, such as natural conditions (hydrology, terrain and geology), environmental conditions, regional economic status, developmental prospects and etc. This article has examined the long stream segment of rivers in the western mountain area and 5 kinds of research methods for the waterway dimension which long stream segment regulation may achieve. They are respectively the optimum waterway dimension of the natural navigation depth or hydraulic permissible navigation depth, the hydraulic optimum waterway dimension, the optimum waterway dimension under the condition of a certain regulation project quantity; the waterway dimension when the regulation project quantity is minimum under a certain annual volume of freight and the optimum waterway dimension under the condition of "limit" increased condition. And the article also proposes specific computation method.

\section{Acknowledgements}

This work was financially supported by National High Technology Research and Development Program 863 (2012AA112508), National Natural Science Foundation of China (51209112), and Basic research funding of national commonweal research institutions (TKS130202).

\section{References}

[1] Zhou Guanlun, Rong Tianfu, Liu Shulun, Lu Hancai et al, Waterway Engineering Manual, People's Communication Press, 2003

[2] Min Chaobin, Research on the Waterway Passing Capacity, Collected Works of Technical Communicating Meeting of Inland River Waterway Regulation Project, People's Communication Press, 1998.12

[3] Zhou Lianyou, a Summary of Formulas about Inland River Waterway Dimension Computation, Port Engineering Technology, 2004, (4): 7-10

[4] Copeland Ronald R, Hall Brad R. Channel restoration hydraulic design procedure. Proceedings of the ASCE Wetlands Engineering River Restoration Conference 1998. ASME, Fairfield, NJ, USA. $6 \mathrm{p}$

[5] McCartney, Bruce L. Optimizing navigation channel dimensions. Proceedings of the Eighteenth Dredging Seminar. Texas A\&M University, Ocean Engineering Program (Report) COE n 284. Publ by Texas A\&M Univ, College Station, TX, USA p 13-20, 1985 